\title{
Evolution of Physicochemical Properties of 2-(2-(4- (4-chloro) phenyl) vinyl)-1, 1, 3-trimethyl-1H-benzo[e] Indolium lodide via Experimental and Quantum Chemical Calculation for Third-Harmonic Generation Applications
}

Eniya Palaniyasan

Periyar University

Anbarasan Radhakrishnan

Periyar University

Shivaraj R. Maidur

HKBK College of Engineering

Parutagouda Shankaragouda Patil

K.L.E. Institute of Technology

Kalyana Sundar Jeyaperumal ( $\sim$ jksundar50@gmail.com )

Periyar University

\section{Research Article}

Keywords: Crystal structure, DFT, Z-scan, Thermal analysis, Third harmonic generation

Posted Date: December 29th, 2021

DOI: https://doi.org/10.21203/rs.3.rs-1184837/v1

License: (a) (1) This work is licensed under a Creative Commons Attribution 4.0 International License.

Read Full License 


\section{Abstract}

In this work, the authors have reported the synthesis, crystal structure, and physicochemical properties of new Indolium based nonlinear optical crystal of 2-(2-(4-(4-chloro) phenyl) vinyl)-1, 1, 3-trimethyl-1Hbenzo[e] Indolium iodide. The title crystal was grown by slow evaporation technique using methanol as a solvent. The harvested crystal is subjected to the single-crystal X-ray diffraction study and found that the title crystal belongs to the monoclinic system with a space group of $\mathrm{P} 2_{1} / \mathrm{n}$. The bandgap and optical window of the material are studied by UV-visible spectroscopic analysis, and the found values are $4.2 \mathrm{eV}$ and $295-800 \mathrm{~nm}$, respectively. The grown crystal is subjected to analyze the thermal stability and found that high-temperature stability value upto $264^{\circ} \mathrm{C}$ and which is sufficient for industrial applications. Furthermore, the effect of temperature on the grown crystal dielectric properties such as dielectric constant and dielectric losses is investigated by impedance spectroscopic analysis. The molecular charge transfer on the material is studied from density functional theory. The various intermolecular interactions existence in the crystal is explored from Hirshfeld surface analysis. Finally, the Z-scan parameters expose that title crystal is a potential material for third-harmonic generation applications. The overall structural studies and functional properties disclose that the grown crystal is well suitable for harmonic generation applications.

\section{Introduction}

Crystals have numerous uses in the present technology, notably in communication and laser technology, optical imaging, optical data storage, sensor, and nonlinear optics. Every day, there are new items have been made and new electronics arise from these crystals. With the advancement of the crystal growth technology of materials with thought-provoking nonlinear aspects, the technological world is looking for new materials. Controlling and considering the mechanisms of crystallization are important in current materials science, specifically it remains an even greater problem in the field of reticulate solids [1-3]. Nonlinear optics (NLO) is the interaction phenomenon with nonlinear crystals that controls the features of immensely coherent light. Advanced technological domains like optoelectronics and photonics depend on the NLO crystals as they are the mandatory functional materials in many applications. The substantial optical nonlinearity is one of the distinctive and crucial qualities of the NLO crystals, because of this attribute, it is being employed as the low profile cut-off frequency. This unique feature of the NLO crystals is employed in photosensitive electronics.

Crystal growers have been made a significant contribution in the last couple of decays to find the high efficient organic NLO materials with outstanding optical characteristics and still, researchers are struggling a lot to find an outstanding material in all aspects such as functional properties as well as growth characteristics and hence still this research topic is considered as a challenging task. Organic crystals are bonded by weak van der Waals interactions and hydrogen bonds [4-6]. Because, the conjugated-bond system is the main framework in these organic NLO materials, and high mobility of the electron density is ascribed to the delocalized distribution of electronic charge owing to orbital overlap. 
Furthermore, the electron donor and acceptor groups on the ends of the bond systems may increase the asymmetric electronic distribution in both ground and excited states, increasing optical nonlinearity.

Compared with the broad measure of examination led on the optical crystals for second-order nonlinear optical applications, the investigation of third-order nonlinear properties of atomic crystal has gotten moderately restricted consideration [7]. The interest in finding new third-order nonlinear optical materials, which are driven essentially by the search of third-order NLO materials for all-optical switching and sensor security applications, are worried about nonlinear absorption (NLA), nonlinear refraction (NLR), and thirdorder nonlinear susceptibility $(x 3)$. The phenomenon of molecular and crystal design techniques for thirdorder NLO applications is of incredible current interest. There have been impressive interest in organic optical material research because of a wide difference of plan prospects, faster nonlinearities that could be utilized for optical modulation, optical switching, and optical memory for emerging optical technologies $[8,9]$. In any case, the enormous large macroscopic nonlinearity is needed for the applications in $\mathrm{THz}$ generation and detection and electro-optics. Additionally, requires different attributes like solubility, crystal growth, melting point, and thermal stability are also essential $[10,11]$.

Especially $D-\pi \bullet \bullet A$ chromophore molecules have exhibited laser frequency conversion, optical communication, electro-optical modulation, THz generation, and detection [12-17]. Currently, developing this type of highly non-linear optical crystal is a challenging task. Therefore, we have selected the heterocyclic benzo [e] indole compounds that have D- $\pi \bullet \bullet A$ chromophore. This heterocyclic benzo [e] indol was an electron acceptor and had a strong electron receptor. The large volume of benzo [e] indol increases the molecular asymmetry, which is highly efficient for nonlinear optical activities. Consequently, efforts were taken to synthesize and grown the new compound, 2-(2-(4-(4-chloro) phenyl) vinyl)-1, 1, 3trimethyl-1H-benzo[e] Indolium iodide (CBI) single crystal. And, we have succeeded to synthesize and develop the best quality of $\mathrm{CBI}$ single crystal with full-scale size $\sim 5 \times 4 \times 1.5 \mathrm{~mm}^{3}$ inside a period of 3 weeks.

In this work, we have grown new benzo [e] indol CBI crystal with large third-order nonlinear properties. The grown crystal is further subjected to various characterization studies of Single-crystal XRD, Optical, Dielectric, and Thermal response of the sample, and the results are discussed to ensure the material properties towards the NLO applications. And the various intermolecular interactions that exist in the $\mathrm{CBI}$ crystal are explored from Hirshfeld surface analysis. To the best of our knowledge, the third-order NLO properties by Z-scan, dielectric, and Thermal studies of $\mathrm{CBI}$ crystals are reported for the first time.

\section{Materials And Methods}

\subsection{Material synthesis and crystal growth}

The equimolar ratio of 1, 1, 2-Trimethyl-1 H-benze[e] indole and lodomethane were dissolved in toluene, and the mixture was refluxed. Then the precipitate was collected by filtration and washed five times with diethyl ether to afford the product as 1, 1, 2, 3-tetramethyl-1H-benzo [e] indol-3-ium-iodide (BI) [18]. The 
product $\mathrm{BI}$ and 4-chlorobenzaldehyde were dissolved in $50 \mathrm{ml}$ of methanol and some amount of piperidine was also added and, which acts as a catalyst. The mixture was heated and refluxed for $18 \mathrm{hrs}$. After the refluxing, the mixture was cooled at ambient temperature. The final product of 2-(2-(4-(4-chloro) phenyl) vinyl)-1, 1, 3-trimethyl-1H-benzo[e] Indolium iodide (CBI) is formed as a solid with an orange color and the grown crystal shown in Fig. 1.

\section{Results And Discussion}

\subsection{Single-crystal X-ray diffraction analysis}

Good quality single crystals of $\mathrm{CBI}$ were selected using an optical polarizing microscope for X-ray diffraction intensity data collection. The X-ray diffraction intensity data has been collected at room temperature from Bruker D8 QUEST ECO Single Crystal X-ray diffractometer equipped with MoKa radiation. The data collection has been monitored by the APEX III program suit. The structure was solved by the direct method with SHELXS2019 and refined by the full-matrix least-squares method with SHELXL2019 implemented in the WINGX2019 package[19, 20]. All H-atoms were located in different Fourier maps and refined isotopically. The molecule with thermal ellipsoid atoms plot was generated from the ORTEP3 incorporated in the WINGX2019 package.

The molecular structure of the $\mathrm{CBI}$ has been determined from the single crystal $\mathrm{x}$-ray diffraction analysis. Fig. 2 shows the $50 \%$ thermal ellipsoid plot of $\mathrm{CBI}$ molecule with the atom numbering scheme. Crystal properties, data collection, and structure refinement details are presented in Table 1. Further, the molecular structure of $\mathrm{CBI}$ has been determined from the gas-phase quantum chemical calculation. The selected geometrical parameters such as bond lengths, bond angles, and torsion angles of CBI molecule determined from the crystal phase and gas phase are collectively presented in Table S1. Comparing the structure of gas-phase and crystal phase, they are found to be equal and same (Fig. 2.); this can be well understood from their torsion angles. In the crystal phase, the intermolecular interactions are analyzed to

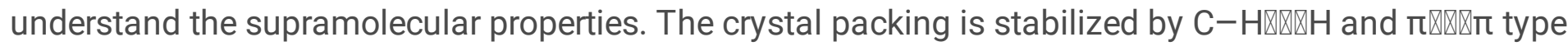

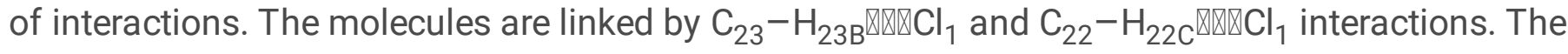
detailed intermolecular interactions with the symmetry code were tabulated in Table 2 and Fig. 3 shows the intermolecular interactions view along the 010 axes and the Crystallographic data for CBI (CCDC Number - 1971339) have been deposited with the Cambridge Crystallographic Data Centre. 
Table 1

Crystal Structure details

\begin{tabular}{|c|c|}
\hline Chemical Formula & $\mathrm{C}_{23} \mathrm{H}_{21} \mathrm{NCl} \mathrm{I}$ \\
\hline Formula Weight (g/mol) & 473.76 \\
\hline Crystal System & Monoclinic \\
\hline Space Group & $\mathrm{P} 2_{1} / \mathrm{n}$ \\
\hline $\mathrm{a} / \AA$ & $7.2849(4)$ \\
\hline $\mathrm{b} / \AA$ & $21.9014(13)$ \\
\hline$c / \AA$ & $12.4896(8)$ \\
\hline $\mathrm{a} /\left(^{\circ}\right)$ & 90.00 \\
\hline$\beta / /\left(^{\circ}\right)$ & $96.359(2)^{\circ}$ \\
\hline $\mathrm{Y} / /\left(^{\circ}\right)$ & 90.00 \\
\hline$V / \AA^{3}$ & $1992.7(2)$ \\
\hline Z & 4 \\
\hline $\mathrm{T} / \mathrm{K}$ & 293 \\
\hline $\mathrm{R}_{1}$ & 0.021 \\
\hline Radiation type & Mo Ka \\
\hline$\mu\left(\mathrm{mm}^{-1}\right)$ & 1.75 \\
\hline Crystal size $\left(\mathrm{mm}^{3}\right)$ & $0.51 \times 0.21 \times 0.15$ \\
\hline \multicolumn{2}{|l|}{ Refinement } \\
\hline$R\left[F^{2}>2 \sigma\left(F^{2}\right)\right], w R\left(F^{2}\right), S$ & $0.037,0.087$ \\
\hline No. of reflections & 3171 \\
\hline No. of parameters & 386 \\
\hline $\mathrm{H}$-atom treatment & $\mathrm{H}$-atom parameters constrained \\
\hline$\Delta \rho_{\max }, \Delta \rho_{\min }\left(\mathrm{e} \AA^{-3}\right)$ & $0.86-1.31$ \\
\hline CCDC No. & 1971339 \\
\hline
\end{tabular}


Table 2

Hydrogen-bond geometry $\left(\AA,{ }^{\circ}\right)$

\begin{tabular}{|lllll|}
\hline$D-\mathrm{H} \cdots \boldsymbol{A}$ & $\boldsymbol{D}-\mathrm{H}$ & $\mathrm{H} \cdots \boldsymbol{A}$ & $\boldsymbol{D} \cdots \boldsymbol{A}$ & $\boldsymbol{D}-\mathrm{H} \cdots \boldsymbol{A}$ \\
\hline $\mathrm{C} 23-\mathrm{H} 23 \mathrm{~B} \cdots \mathrm{Cl} 1$ & 0.96 & 3.81 & 2.961 & 148 \\
\hline $\mathrm{C} 22-\mathrm{H} 22 \mathrm{C} \cdots \mathrm{Cl} 1$ & 0.96 & 3.60 & 2.914 & 128 \\
\hline
\end{tabular}

\section{2 Optical Studies}

For this study, a CBI solution was dissolved in methanol. Most Indolium compounds are soluble in methanol, since this causes the state to dissociate, resulting in free cations and anions. Fig. 4 depicts the measured optical absorption spectra of CBI. We can estimate the optical band gap and investigate the nature of transitions using absorption peaks. The spectrum shows a higher absorption peak at $295 \mathrm{~nm}$, which corresponds to the $n-\pi$ electronic transition. The calculated bandgap was to be $4.2 \mathrm{eV}$. Because of its outstanding optical behavior, absorption is not noticed between 295 and $800 \mathrm{~nm}$, which is a benefit of $\mathrm{CBI}$ and an essential criterion for materials employed in nonlinear optical applications.

\subsection{Thermal Stability}

Thermal stability of the CBI crystals characterized via differential thermal analysis (DTA) and thermal gravimetric (TG) measurements are shown in Fig. 5. The DTA and TG of the grown crystal were recorded in the range of 30-600 $\otimes C$ under $\mathrm{N}_{2}$ atmosphere using the instrument Model Q600 SDT and Q20. As shown in Fig. 5 , the sharpness of the endothermic peak at $264^{\circ} \mathrm{C}$ suggests the melting point of the $\mathrm{CBI}$ crystal. Interestingly, this is a higher melting temperature than that of benchmark organic NLO crystals of $\mathrm{OH} 1\left(212^{\circ} \mathrm{C}\right)$ and DAST $\left(256^{\circ} \mathrm{C}\right)$. The $\mathrm{CBI}$ was stable up to $202 \otimes \mathrm{C}$, and also, the CBI exhibited very high

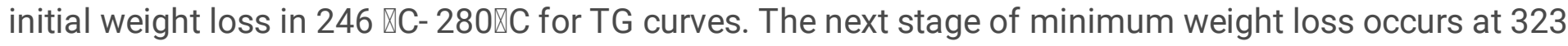
${ }^{\circ} \mathrm{C}-423{ }^{\circ} \mathrm{C}$. The following weight loss is the complete decomposition beyond the temperature of $438^{\circ} \mathrm{C}$. Finally from this DTA-TG analysis, the thermal stability of the material is higher than the benchmark of nonlinear materials of DAST and $\mathrm{OH} 1$, and hence it can be exploited for optoelectronic device applications up to $264^{\circ} \mathrm{C}$ and suitable for high physicochemical stability applications. This advantage is also suitable for stable high-power Terahertz output.

\subsection{Dielectric analysis}

By the way, in the NLO materials research, the investigation of temperature depends on dielectric properties, and its stability concerning temperature is highly crucial. The degree of the polarization greatly contributes to the harmonic generation of the crystal [21-23]. Based on the technological importance, we have performed impedance spectroscopic studies using an impedance analyzer to understand the test specimen's electronic polarization. The calculated dielectric constant and dielectric loss profiles over the frequency region $1 \mathrm{~Hz}$ to $1 \mathrm{MHz}$ concerning various temperature are provided in Fig. 6 (a) and Fig. 6 (b). As seen in Fig. 1, at $40^{\circ} \mathrm{C}$ the constant dielectric profile showed the universal behavior of the previous NLO 
materials concerning the frequency, and it illustrated that the four types of polarizations are contributed $[24,25]$.

Furthermore, we increased the temperature to 60,80 , and $100^{\circ} \mathrm{C}$ and obtained constant dielectric profiles are presented in Fig. 6 (a) and Fig. 6 (b). Surprisingly, we could not see any changes in the dielectric constant values, and the dielectric constant trend is also similar to all the other temperatures. Hence, it is clear that the electronic polarization of the test sample with respect to temperature is not affected, and the applied temperature has not induced any surface and structural defects, and the resultant output of the harmonic generation is not affected. Note that such electronic polarization stability is much required for the electronic devices fabrications to save the device by the external parameters [26]. In addition to that, based on previous reports, numbers of NLO materials have been undergoing significant changes in the constant dielectric profiles concerning temperature. Herewith, few reports are discussed based on their changes in the dielectric constant profiles to temperature for better understanding the importance of the stability of the dielectric properties. Sangeetha et al., have been reported glycinium hydrogen fumarate glycine solvate monohydrate single crystal dielectric constant profiles concerning temperature and found massive changes in the dielectric constant and loss [27]. L-Threonine formate crystal dielectric constant profiles with respect to temperatures have been reported by Redrothu Hanumantharao et al. and found considerable changes in the dielectric constant with respect to temperature [28] and similar kind of results are also found in L-Asparaginium picrate [29] and L-Argininium perchlorate [30]. Nevertheless, in the present case, the tilted crystal does not show considerable change with respect to temperature. Hence, the titled material is highly deserved for the device fabrications under different temperatures.

\subsection{Hirshfeld surface analysis}

Hirshfeld surface analysis is used to visualize and quantify various types of intermolecular interactions in the crystal environment. Also, it is used to define the partitioning of electron density of the crystal into molecular fragments[31, 32]. Intermolecular contacts and their impact on crystal packing were analyzed using Crystallographic Information File (CIF) in Crystal Explorer 17.5 software [33, 34]. The $\mathrm{d}_{\mathrm{e}}$ (outside nuclei surface) and $d_{i}$ (inside surface) were scaled in different colors and displayed the sum in Fig. 7(a) and 7 (b) (vdw radii in white, close-contact interactions in red and short contacts than vdw radii in blue surfaces). Further, the shaped index explores the $\mathrm{C}-\mathrm{H} \bullet \bullet \bullet \pi$ and $\pi \bullet \bullet \pi$ interactions. Fingerprint plots used to determine the contact distances ( $\mathrm{di}, \mathrm{de}$ ) and the reciprocal contacts are in the range from 0.6 to $2.8 \AA$. In the $d_{\text {norm }}$ plot (Fig. 7), white surface is the sum of vdw radii, the blue surface is the short contact distances than vdw radii and red surface explains the hydrogen bond contacts.

Further, the intermolecular interactions of the molecule in the crystal can be characterized by the 2D shape of fingerprint plots shown in Fig. 8. The figure displays the fingerprint plot, which helps quantify the contribution of different types of interactions in the solid-state. The $\mathrm{H} \cdots \mathrm{H}, \mathrm{O} \cdots \mathrm{H}$ and $\mathrm{C} \cdots \mathrm{H}$ type of interactions are highly presented in $\mathrm{CBI}$, which confirm that the Vander Walls and $\mathrm{C}-\mathrm{H} \cdots \pi$ interactions play the major role in the crystal environment. The $\mathrm{C} \cdots \mathrm{H}$ fingerprint plots show similar symmetrical wing shape, the $\mathrm{O} \cdots \mathrm{H}$ plot looks like sharp spikes and the $\mathrm{H} \cdots \mathrm{H}$ plot are overlapped in full fingerprint plots that 
would commonly separate from each other. Therefore, this fingerprint plot confirms that weaker hydrogen bonding leads to the stability of the compound in the crystal phase.

\subsection{Frontier Molecular Orbital analysis}

Geometry optimization of the synthesized molecule (CBI) was carried out by Becke's three-parameter hybrid exchange functional (B3LYP) level of density functional theory (DFT). All the atoms were optimized by the LANL2DZ basis set to get more accurate energy optimization of the molecule, all the gas phase structural optimization and computation were performed by the Gaussian09 software package. The higher occupied molecular orbital (HOMO), lower unoccupied molecular orbital (LUMO) have been analyzed from Gauss view package [35]. The essential quantum chemical descriptors such as highest occupied molecular orbital (HOMO) and lowest unoccupied molecular orbital (LUMO) were calculated for the imperator in the molecule, which is highly related to the chemical and biological activities. The molecular surface of HOMO and LUMO maps of both complexes were drawn at 0.2 au and shown in Fig.9, in which the HOMO and LUMO energy surfaces were found over the keto group and centre ring regions, respectively. The positive and negative regions are shown in green and red colors, respectively. The global reactivity descriptors were calculated to understand the quantum chemical properties. The calculated values for the HOMO and LUMO energies are $-4.76 \mathrm{eV}$ and $-3.16 \mathrm{eV}$, respectively. The bandgap is $1.60 \mathrm{eV}$. The calculated electron affinity, ionization potential, and electronegativity are $3.16,4.76$, and $3.96 \mathrm{eV}$. The electron affinity and electronegativity values are low compared to the ionization potential, which suggests the molecule has high reactivity.

\subsection{Nonlinear optical property}

The third-order NLO properties of CBI were studied using the open and closed aperture Z-scan technique [36]. It is far a sensitive and straightforward method to measure the optical nonlinearities with high accuracy. This method focuses on quantifying NLA (nonlinear absorption) and NLR (nonlinear refraction) simultaneously with a single experiment. It gives the most helpful information about the sign and magnitude of the obtained nonlinearities. Open aperture (OA) Z-scan is used to determine NLA and closed aperture (CA) Z-scan to Measure NLR. In this current work, we achieved Z-scan measurements with a diode-pumped solid-state (DPSS) continuous wave (CW) laser of Gaussian beam profile with $532 \mathrm{~nm}$ and $200 \mathrm{~mW}$ output power. The laser beam becomes tightly targeted, with the Plano-convex lens having a $28.6 \mathrm{~cm}$ focal length. The beam size and the depth of the laser at the focus point are measured to be 33 $\mu \mathrm{m}$ and $12.8 \mathrm{~kW} / \mathrm{cm}^{2}$, respectively. The $1 \mathrm{~mm}$ cuvette containing the $\mathrm{CBI}$ solution was placed on a stepper motor (100 $\mu \mathrm{m}$ resolution) and scanned across the lens's focus along the Z-axis path (laser beam propagation). The Z-scan measurement of the thin medium situation is fulfilled because sample thickness is much less than the Rayleigh length $\left(Z_{0}=6.15 \mathrm{~mm}\right)$. The cross-section of the laser beam coming out of the sample turned into controlled to placing an aperture of variable diameter within a farfield discipline. Whereas only in part transmitted intensity is measured in closed aperture experiment, and the aperture size became kept at $40 \%$ of the linear transmittance $(S=0.4)$. The detectors had been connecting to the virtual electric control unit, the interpretation stage and data acquisition changed into control with the aid of the automatic Z-scan program 


\subsubsection{Nonlinear absorption}

It is determined that $\mathrm{CB}$ I suggests a lower transmittance with an increase in intensity, indicating the reverse saturable absorption (RSA) behavior, i.e., the absorption is maximum at the focus, wherein the intensity is maximum $(Z=0)$. Therefore, a signal of the nonlinear absorption is positive for $R S A(\beta>0)$. In order to estimate the nonlinear absorption coefficients quantitatively and affirm the corresponding NLA mechanism, we equipped the experimental open aperture data with the theoretical NLA model. The stable lines within Fig. 10 represent the theoretical curves, and open circles are experimental information. The Two-photon absorption (TPA) kind of nonlinear absorption becomes determined to be the fine approximation. The normalized transmission as a feature of the sample position is given by [21].

$$
T(Z)=1-\frac{\beta I_{0} L_{e f f}}{2 \sqrt{2}\left(1+Z^{2} / Z_{0}^{2}\right)}
$$

1

Where $\beta$ is the two-photon absorption coefficient, which is found to be within the order $\sim 10^{-4} \mathrm{cmW}^{-1}$ for all samples. $L_{e f f}=\left(1-\exp ^{-a L}\right) / a$, is the effective length of the sample, $a$ is the linear absorption coefficient, and $L$ is the thickness of the sample. $I_{0}$ is the intensity of the laserbeam at the focus. The corresponding $\beta$ values of the samples are supplied in Table 3 .

Figure 10: Nonlinear absorption curves of CBI

\subsubsection{Nonlinear refraction}

The nonlinear refraction curves of the $\mathrm{CBI}$ are shown in Fig. 11. We observed negative nonlinear refraction behavior for both the samples due to the self-focusing effect of the samples. The nonlinear phase shift of the samples was obtained by fitting the closed aperture data with the following relation [37].

$$
T(Z)=1-\frac{\left(4 X ?^{?}{ }_{0}\right)}{\left(X^{2}+1\right)\left(X^{2}+9\right)}
$$

2

where $T(Z)$ is the normalized transmittance, $\Delta \backslash$ upvarphi $_{0}$ is the nonlinear phase shift, and $X=Z / Z_{0}$. Nonlinear refractive index $n_{2}$ can be calculated by using the $\Delta \backslash$ upvarphi ${ }_{0}$ value in the relation ? ? ${ }_{0}=k n_{2} I_{0} L_{\text {eff }}$ where $k(=2 \pi / \lambda)$ is the wave vector. The obtained values of the NLR coefficients are found to be in the order $\sim 10^{-8} \mathrm{~cm}^{2} \mathrm{~W}^{-1}$ and are given in Table 3 .

The third-order nonlinear refraction and absorptions are directly related to the real and imaginary parts of the third-order nonlinear optical susceptibility, respectively. The real, imaginary, and magnitude of the susceptibility of the $\mathrm{CBI}$ and $\mathrm{HBI}$ have been decided with using the following relations [38]., 


$$
?^{(3)}=?_{R}^{(3)}+i ?_{I}^{(3)}
$$

$$
?_{R}^{(3)}(e s u)=\frac{c n_{0}^{2}}{120 p^{2}} n_{2}\left(m^{2} / W\right)
$$

4

$$
\chi_{I}^{(3)}(e s u)=\frac{c^{2} n_{0}^{2}}{240 \Pi^{2} \omega} \beta(m / W)
$$

5

Where $\mathrm{c}$ is the velocity of light, $\mathrm{n}_{0}$ is the linear refractive index and $\omega$ is the angular frequency of the incident photons. The obtained magnitude of the NLO susceptibility of CBI is in the order $\sim 10^{-7}$ esu. Further, the molecular second hyperpolarizability (microscopic polarizability) of the samples was also calculated and the values are found to be in the order $\sim 10^{-26} \mathrm{esu}$, indicates the acquired values of nonlinear optical parameters from Z scan measurements of CBI crystal. The comparison of third-order optical susceptibility of $\mathrm{CBI}$ crystal with a few well known organic crystals is given in Table.4. The grown $\mathrm{CBI}$ has a very high susceptibility, which also increases the capability of the material in NLO tool fabrication.

Table 3

Nonlinear optical parameter for CBI Crystals

\begin{tabular}{|ll|}
\hline$\Delta \boldsymbol{\phi}$ & 0.45 \\
\hline Nonlinear absorption coefficient $(\beta)$ & $4.80 \times 10^{-4}(\mathrm{~cm} / \mathrm{w})$ \\
\hline Nonlinear refractive index $\left(\mathrm{n}_{2}\right)$ & $-0.33 \times 10^{-8}\left(\mathrm{~cm}^{2} / \mathrm{W}\right)$ \\
\hline Real part of third order susceptibility $\left(\operatorname{Re} \chi^{(3)}\right)$ & $-1.74 \times 10^{-7} \mathrm{esu}$ \\
\hline The imaginary part of third-order susceptibility $\left(\operatorname{Im} \chi^{(3)}\right)$ & $1.08 \times 10^{-7} \mathrm{esu}$ \\
\hline Third-order nonlinear susceptibility $\left(\chi^{(3)}\right)$ & $2.05 \times 10^{-7} \mathrm{esu}$ \\
\hline Second order molecular hyperpolarizability $\left(\mathrm{Y}_{\mathrm{h}}\right)$ & $0.97 \times 10^{-26} \mathrm{esu}$ \\
\hline
\end{tabular}


Table 4

Comparison of $\chi 3$ value of CBI with some other NLO single crystals

\begin{tabular}{|llll|}
\hline S. No & Crystals & Third-order optical susceptibility $(\chi 3)$ & References \\
\hline 1. & VMST & $9.69 \times 10^{-12}$ esu & {$[39]$} \\
\hline 2. & 2 A5NPBr & $6.76 \times 10^{-09} \mathrm{esu}$ & {$[40]$} \\
\hline 3. & DSMOS & $5.05 \times 10^{-8} \mathrm{esu}$ & {$[41]$} \\
\hline 4. & $3 \mathrm{ETSI}$ & $3.84 \times 10^{-7} \mathrm{esu}$ & {$[42]$} \\
\hline 5. & CBI & $2.05 \times 10^{-7} \mathrm{esu}$ & Present Work \\
\hline
\end{tabular}

\section{Conclusion}

The new organic compound of 2-(2-(4-(4-chloro) phenyl) vinyl)-1, 1, 3-trimethyl-1H-benzo[e] Indolium iodide was synthesized and the crystal was grown by slow evaporation technique. The structural features are established from a single-crystal X-ray diffraction analysis. As per the single XRD data, the CBI crystal belongs to the monoclinic crystal system with the centrosymmetric symmetry space group of $P Z_{1} / \mathrm{n}$. The optical absorption study reveals that the lower absorption in the visible spectral region is a good fit for the NLO applications. The material is thermally stable upto $264^{\circ} \mathrm{C}$, which is higher than the typical DAST crystal. The molecular level charge transfer of the material was studied from the frontier molecular orbital analysis. At various temperatures, the fluctuation in the dielectric constant was investigated. Z-scan with a closed aperture showed the positive nonlinear absorption coefficient. According to the Z-Scan test, the crystal nonlinear refractive index is in the range of $10^{-8} \mathrm{~cm}^{2} / \mathrm{W}$, using $532 \mathrm{~nm}$ laser pulses. As overall results of the characterizations of the $\mathrm{CBI}$ crystal, we can conclude that the grown crystal may be employed as an effective NLO material.

\section{References}

[1] J.Z. D.S. Chemla, Nonlinear Optical Properties of Organic Molecules and Crystals, 1 (1987).

[2] D.J.W. P. N. Prasad, Introduction to Nonlinear Optical Effect in Molecules and Polymers, (1991).

[3] M. Manivannan, S.A. Martin Britto Dhas, M. Jose, Ferroelectric Behavior of Organic Terahertz Radiating DAST Crystal, J. Inorg. Organomet. Polym. Mater. 27 (2017) 1870-1877.

https://doi.org/10.1007/s10904-017-0655-0.

[4] J. Zyss, Molecular Nonlinear Optics: Materials Physics and Devices, (1993).

[5] D.J. (Ed. ). J. Badan, R. Hierle, A. Perigand, J. Zyss, In: and Williams, Nonlinear Optical Properties of Organic Molecules and Polymeric Materials, (1993). 
[6] M. Samoc, N. Gauthier, M.P. Cifuentes, F. Paul, C. Lapinte, M.G. Humphrey, Electrochemical Switching of the Cubic Nonlinear Optical Properties of an Aryldiethynyl-Linked Heterobimetallic Complex between Three Distinct States, Angew. Chemie Int. Ed. 45 (2006) 7376-7379.

https://doi.org/10.1002/anie.200602684.

[7] D.J.W. P.N. Prasad, Introduction to Nonlinear Optical Effects in Organic Molecules and Polymers, Wiley. (1991).

[8] Z. Latajka, G. Gajewski, A.J. Barnes, D. Xue, H. Ratajczak, Hyperpolarizabilities of some model hydrogen-bonded complexes: PM3 and ab initio studies, J. Mol. Struct. 928 (2009) 121-124. https://doi.org/10.1016/j.molstruc.2009.03.024.

[9] X. Gu, D. Xue, H. Ratajczak, Crystal engineering of lanthanide-transition-metal coordination polymers, J. Mol. Struct. 887 (2008) 56-66. https://doi.org/10.1016/j.molstruc.2007.11.052.

[10] D. Rezzonico, S.-J. Kwon, H. Figi, O.-P. Kwon, M. Jazbinsek, P. Günter, Photochemical stability of nonlinear optical chromophores in polymeric and crystalline materials, J. Chem. Phys. 128 (2008) 124713. https://doi.org/10.1063/1.2890964.

[11] J. Crasta, V., Ravindrachary, V., Lakshmi, S., Pramod, S. N., Shridar, M. A., \& Shashidhara Prasad, Growth, characterization and crystal structure analysis of 1-(4-chlorophenyl)-3-(4-chlorophenyl)-2-propen1-one., J. Cryst. Growth. 275(1-2) (2005) e329-e335. https://doi.org/10.1016/j.jcrysgro.2004.10.11.

[12] W. Guan, G. Yang, C. Liu, P. Song, L. Fang, L. Yan, Z. Su, Reversible Redox-Switchable Second-Order Optical Nonlinearity in Polyoxometalate: A Quantum Chemical Study of [PW 11039 (ReN)] n - (n= 3-7), Inorg. Chem. 47 (2008) 5245-5252. https://doi.org/10.1021/ic8001527.

[13] M. Jazbinsek, U. Puc, A. Abina, A. Zidansek, Organic Crystals for THz Photonics, Appl. Sci. 9 (2019) 882. https://doi.org/10.3390/app9050882.

[14] L.T. Cheng, W. Tam, S.H. Stevenson, G.R. Meredith, G. Rikken, S.R. Marder, Experimental investigations of organic molecular nonlinear optical polarizabilities. 1. Methods and results on benzene and stilbene derivatives, J. Phys. Chem. 95 (1991) 10631-10643. https://doi.org/10.1021/j100179a026.

[15] L.R. Dalton, P.A. Sullivan, D.H. Bale, Electric Field Poled Organic Electro-optic Materials: State of the Art and Future Prospects, Chem. Rev. 110 (2010) 25-55. https://doi.org/10.1021/cr9000429.

[16] C. Bosshard, K. Sutter, P. Prêtre, J. Hulliger, M. Flörsheimer, P. Kaatz, P. Günter, Organic Nonlinear Optical Materials, CRC Press, 2020. https://doi.org/10.4324/9780429114090.

[17] C.C. Evans, M. Bagieu-Beucher, R. Masse, J.-F. Nicoud, Nonlinearity Enhancement by Solid-State Proton Transfer: A New Strategy for the Design of Nonlinear Optical Materials, Chem. Mater. 10 (1998) 847-854. https://doi.org/10.1021/cm970618g. 
[18] H. Chen, Q. Ma, Y. Zhou, Z. Yang, M. Jazbinsek, Y. Bian, N. Ye, D. Wang, H. Cao, W. He, Engineering of Organic Chromophores with Large Second-Order Optical Nonlinearity and Superior Crystal Growth Ability, Cryst. Growth Des. 15 (2015) 5560-5567. https://doi.org/10.1021/acs.cgd.5b01216.

[19] G.M. Sheldrick, A short history of SHELX, Acta Crystallogr. Sect. A Found. Crystallogr. 64 (2008) 112122. https://doi.org/10.1107/S0108767307043930.

[20] J. Lübben, C. Volkmann, S. Grabowsky, A. Edwards, W. Morgenroth, F.P.A. Fabbiani, G.M. Sheldrick, B. Dittrich, On the temperature dependence of $\mathrm{H}-\mathrm{U}$ iso in the riding hydrogen model, Acta Crystallogr. Sect. A Found. Adv. 70 (2014) 309-316. https://doi.org/10.1107/S2053273314010626.

[21] S.R. Maidur, P.S. Patil, Z-scan studies of third-order nonlinear optical and optical limiting properties of chalcones doped Poly(methyl methacrylate) thin films for visible laser protection, Opt. Mater. (Amst). 84 (2018) 28-37. https://doi.org/10.1016/j.optmat.2018.06.050.

[22] B. Behera, P. Nayak, R.N.P. Choudhary, Structural and impedance properties of KBa2V5015 ceramics, Mater. Res. Bull. 43 (2008) 401-410. https://doi.org/10.1016/j.materresbull.2007.02.042.

[23] P. Khatri, B. Behera, R.N.P. Choudhary, Structural and impedance properties of Ca3Nb208 ceramics, J. Phys. Chem. Solids. 70 (2009) 385-389. https://doi.org/10.1016/j.jpcs.2008.11.006.

[24] P.K. and P.M. P. Balamurugaraj, S. Suresh, Growth, Optical, Mechanical, Dielectric and Photoconductivity Properties of L-Proline Succinate NLO Single Crystal, J. Mater. Phys. Chem. 1 (2013) 4-8. https://doi.org/10.12691/jmpc-1-1-2.

[25] A. Eroğlu, A. Tataroğlu, Ş. Altındal, On the temperature-dependent dielectric properties, conductivity, and resistivity of MIS structures at 1MHz, Microelectron. Eng. 91 (2012) 154-158. https://doi.org/10.1016/j.mee.2011.07.016.

[26] B. Babu, J. Chandrasekaran, B. Mohanbabu, Y. Matsushita, M. Saravanakumar, Growth, physicochemical and quantum chemical investigations on 2-amino 5-chloropyridinium 4carboxybutanoate - an organic crystal for biological and optoelectronic device applications, RSC Adv. 6 (2016) 110884-110897. https://doi.org/10.1039/C6RA15791B.

[27] P. Sangeetha, P. Jayaprakash, P. Ramesh, S. Sudha, G. Vinitha, M. Nageshwari, M.L. Caroline, Crystal growth, spectroscopic, optical, thermal and hirshfeld surface analysis of glycinium hydrogen fumarate glycine solvate monohydrate (GHFGSM): A third harmonic nonlinear optical organic crystal, J. Mol. Struct. 1213 (2020) 128187. https://doi.org/10.1016/j.molstruc.2020.128187.

[28] R. Hanumantharao, S. Kalainathan, Growth, spectroscopy, dielectric and nonlinear optical studies of novel organic NLO crystal: I-Threonine formate, Spectrochim. Acta Part A Mol. Biomol. Spectrosc. 94 (2012) 78-83. https://doi.org/10.1016/j.saa.2012.03.062. 
[29] A. P. Srinivasan, T. Kanagasekaran, R. Gopalakrishnan, G. Bhagavannarayana, P. Ramasamy, Studies on the Growth and Characterization of L-Asparaginium Picrate (LASP)sA Novel Nonlinear Optical Crystal, Cryst. Growth Des. 6 (2006) 1663-1670. https://doi.org/10.1021/cg060094+.

[30] S. Aruna, G. Bhagavannarayana, M. Palanisamy, P.C. Thomas, B. Varghese, P. Sagayaraj, Growth, morphological, mechanical and dielectric studies of semi-organic NLO single crystal: I-argininium perchlorate, J. Cryst. Growth. 300 (2007) 403-408. https://doi.org/10.1016/j.jcrysgro.2006.11.296.

[31] M.A. Spackman, D. Jayatilaka, Hirshfeld surface analysis, CrystEngComm. 11 (2009) 19-32. https://doi.org/10.1039/B818330A.

[32] M.C. Etter, Hydrogen bonds as design elements in organic chemistry, J. Phys. Chem. 95 (1991) 46014610. https://doi.org/10.1021/j100165a007.

[33] P.R.S. M.J. Turner, J.J. McKinnon, S.K. Wolf, D.J. Grimwood, CrystalExplorer17, (2017).

[34] H. Wang, H. Xiao, N. Liu, B. Zhang, Q. Shi, Three New Compounds Derived from Nitrofurantoin: X-Ray Structures and Hirshfeld Surface Analyses, Open J. Inorg. Chem. 05 (2015) 63-73. https://doi.org/10.4236/ojic.2015.53008.

[35] R. Anbarasan, P. Eniya, M. Anna Lakshmi, J. Kalyana Sundar, Structural, spectral, optical, thermal and quantum chemical investigations on ethylenediammonium tetrachloro zinc crystal for optoelectronic applications, J. Mol. Struct. 1188 (2019) 165-172. https://doi.org/10.1016/j.molstruc.2019.03.091.

[36] E.W.V.S. M. Sheik-Bahae, A.A. Said, T.H. Wei, D.J. Hagan, Sensitive measurement of optical nonlinearities using a single beam, IEEE J. Quantum Electron. 26 (1990) 760-769.

[37] M. Sheik-bahae, A.A. Said, E.W. Van Stryland, High-sensitivity, single-beam n_2 measurements, Opt. Lett. 14 (1989) 955. https://doi.org/10.1364/OL.14.000955.

[38] S.R. Maidur, J.R. Jahagirdar, P.S. Patil, T.S. Chia, C.K. Quah, Structural characterizations, Hirshfeld surface analyses, and third-order nonlinear optical properties of two novel chalcone derivatives, Opt. Mater. (Amst). 75 (2018) 580-594. https://doi.org/10.1016/j.optmat.2017.11.008.

[39] M. Krishna Kumar, S. Sudhahar, P. Pandi, G. Bhagavannarayana, R. Mohan Kumar, Studies of the structural and third-order nonlinear optical properties of solution grown 4-hydroxy-3-methoxy-4'-N'methylstilbazolium tosylate monohydrate crystals, Opt. Mater. (Amst). 36 (2014) 988-995. https://doi.org/10.1016/j.optmat.2014.01.007.

[40] S. Vediyappan, R. Arumugam, K. Pichan, R. Kasthuri, S.P. Muthu, R. Perumal, Crystal growth and characterization of semi-organic 2-amino-5-nitropyridinium bromide (2A5NPBr) single crystals for thirdorder nonlinear optical (NLO) applications, Appl. Phys. A. 123 (2017) 780. https://doi.org/10.1007/s00339-017-1394-3. 
[41] A. Antony Raj, R. Gunaseelan, P. Sagayaraj, Investigation on third-order nonlinear optical, electrical, and surface properties of organic stilbazolium crystal of 4-N,N-dimethylamino-N'-methylstilbazolium pmethoxybenzenesulfonate, Opt. Mater. (Amst). 38 (2014) 102-107. https://doi.org/10.1016/j.optmat.2014.10.011.

[42] K. Nivetha, W. Madhuri, S. Kalainathan, Synthesis, growth, crystal structure and characterization of new stilbazolium derivative single crystal: (E)-4-(3-ethoxy-2-hydroxystyryl)-1-methyl pyridinium iodide (3ETSI), J. Mater. Sci. Mater. Electron. 28 (2017) 8937-8949. https://doi.org/10.1007/s10854-017-66240 .

\section{Figures}

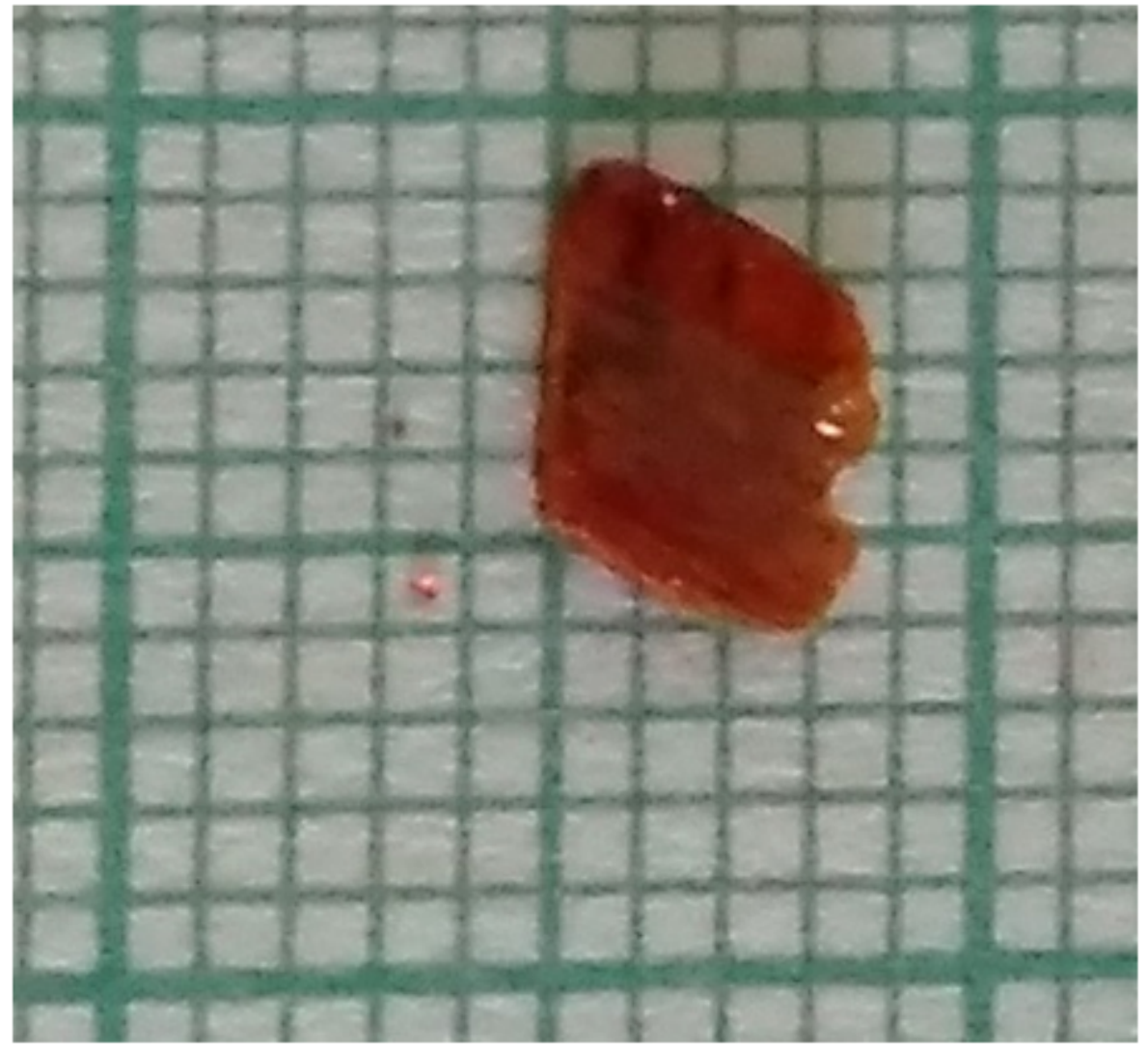

\section{Figure 1}

Photograph of as grown CBI crystals 


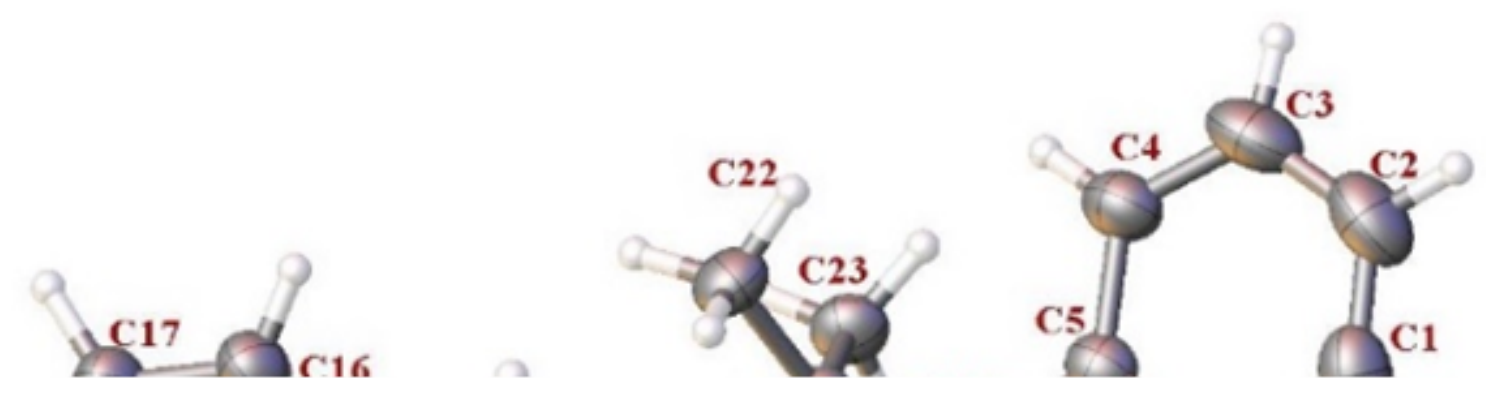

Figure 2

Ortep view of $\mathrm{CBI}$ molecule

0

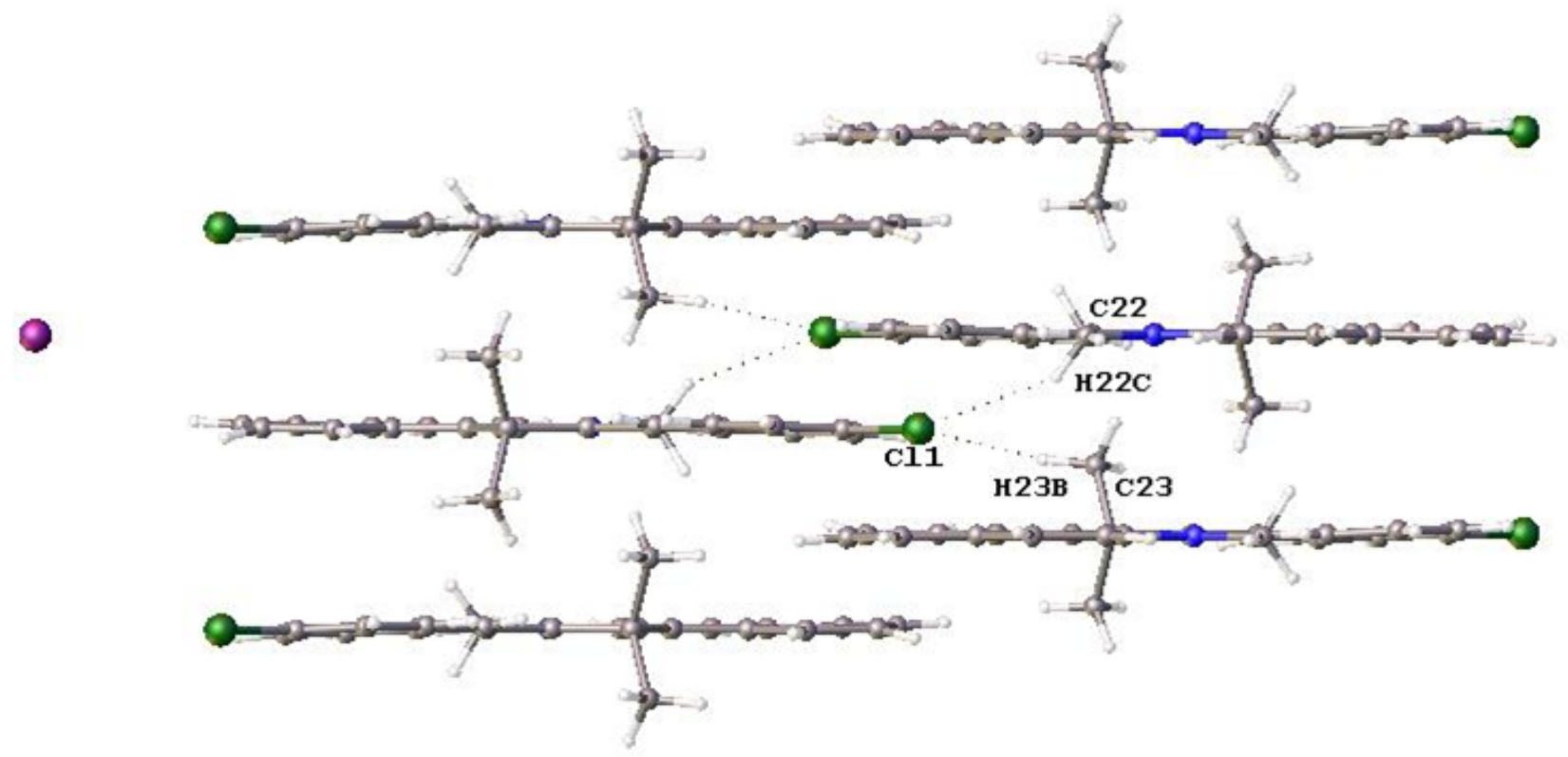

Figure 3 
Intermolecular interactions view along 010 axis

Figure 4

Optical absorption spectra of CBI

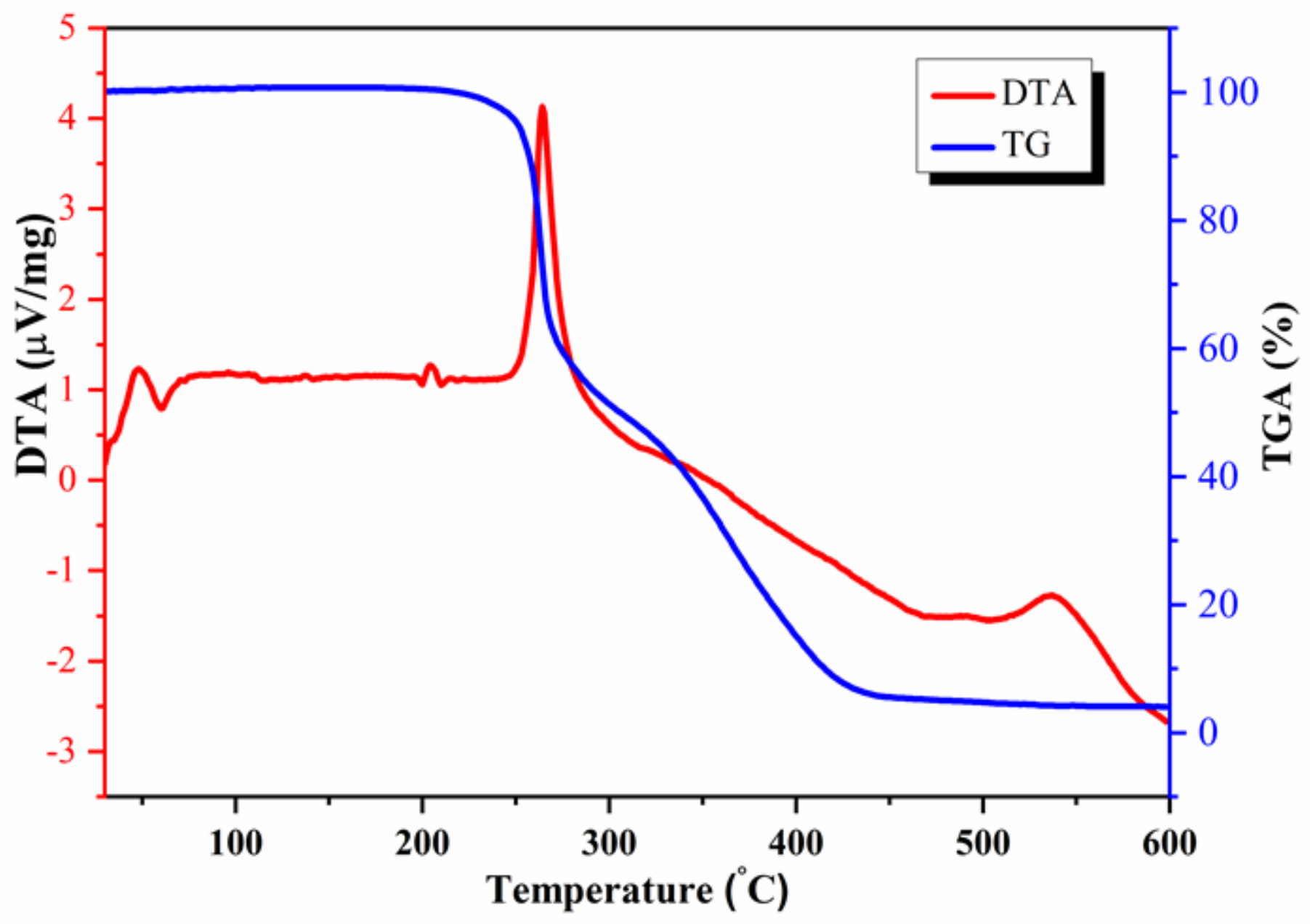

Figure 5

TGA and DTA analysis of CBI

Figure 6

(a) Dielectric constant with respect to temperature and (b) Dielectric Loss with respect to temperature 

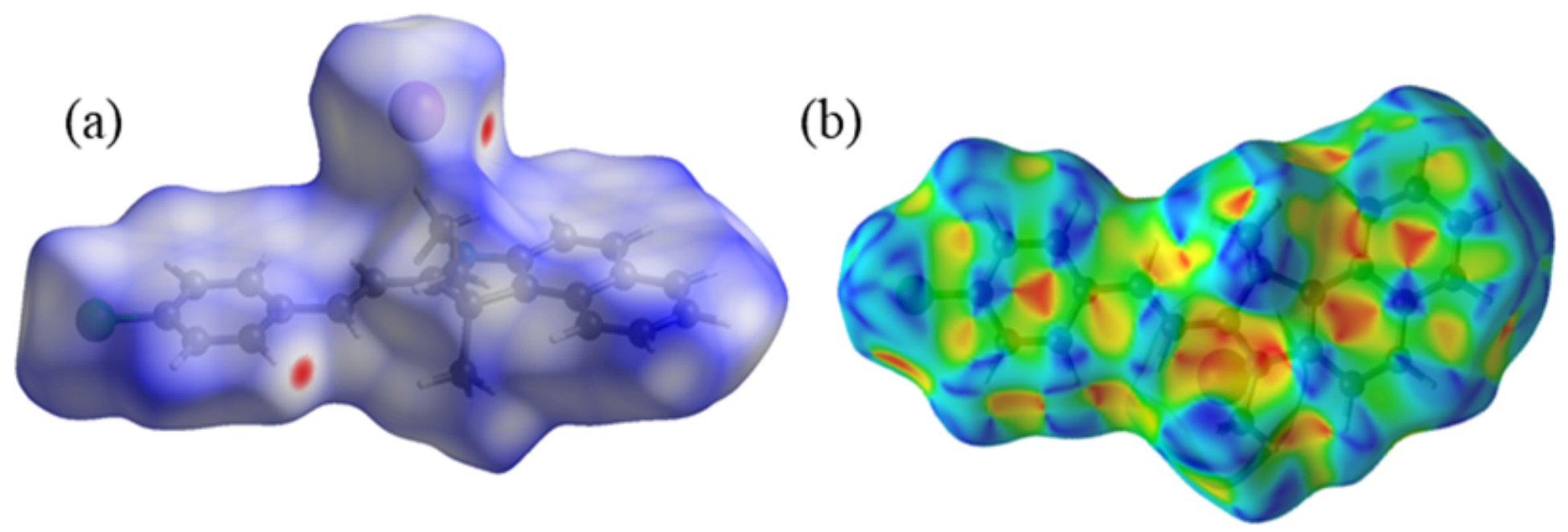

Figure 7

Hirshfeld surface of a) dnorm structure b) shape index structure of $\mathrm{CBI}$ molecule
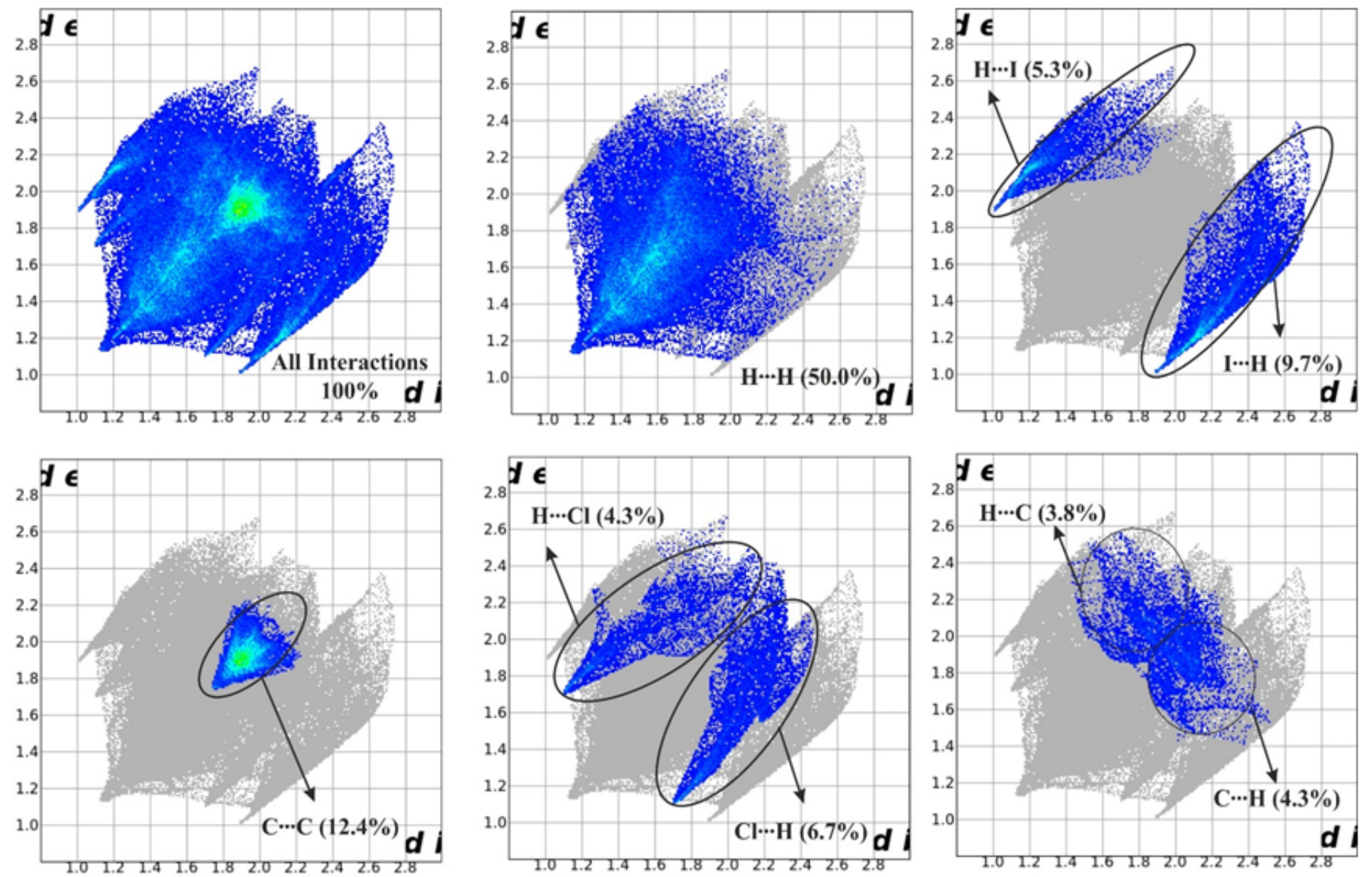

Figure 8 


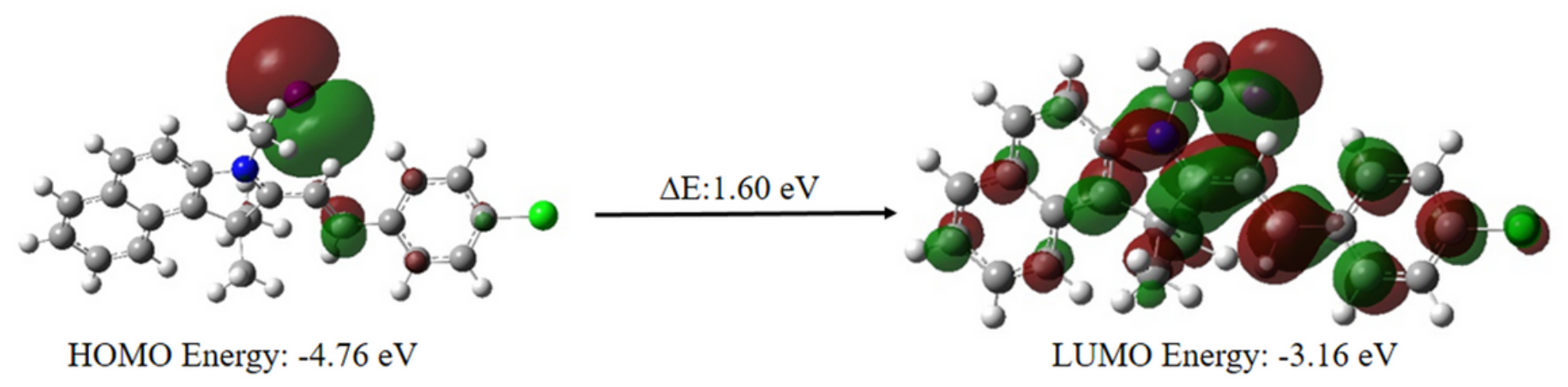

Figure 9

The HOMO and LUMO has drawn at 0.02 au level

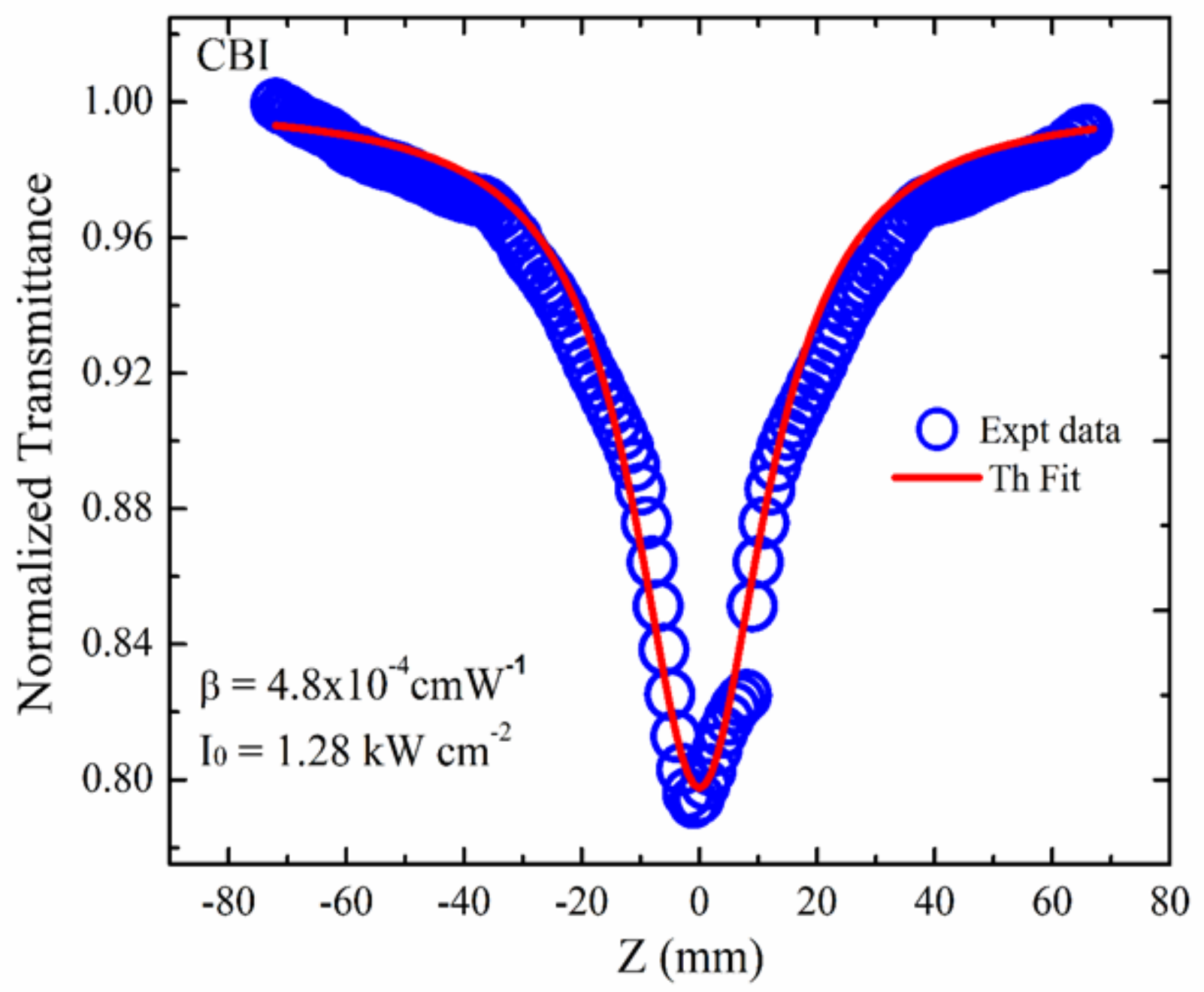


Figure 10

Nonlinear absorption curves of CBI

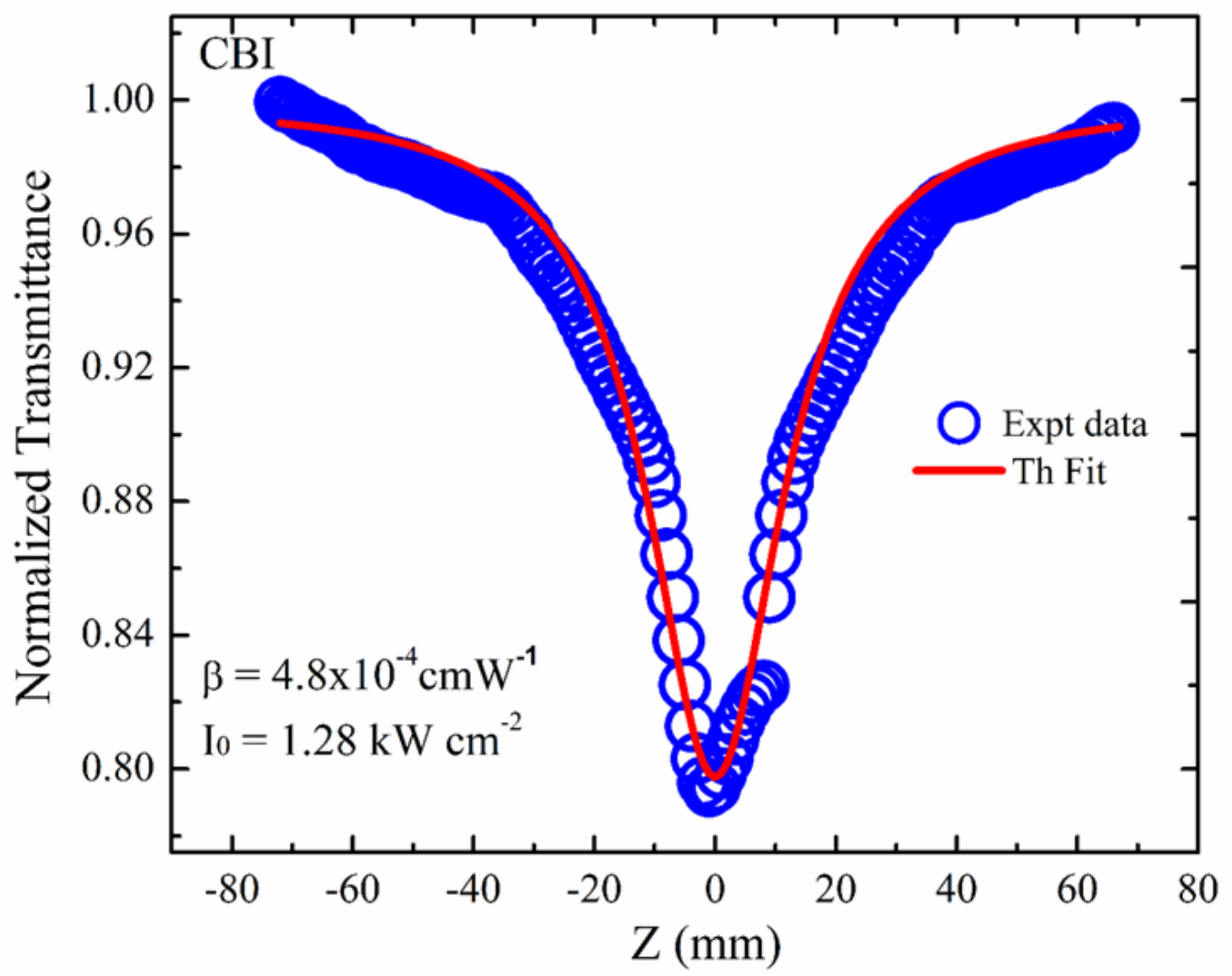

Figure 11

Nonlinear refraction curves of $\mathrm{CBI}$

\section{Supplementary Files}

This is a list of supplementary files associated with this preprint. Click to download.

- SupplymentryTableS1.docx 